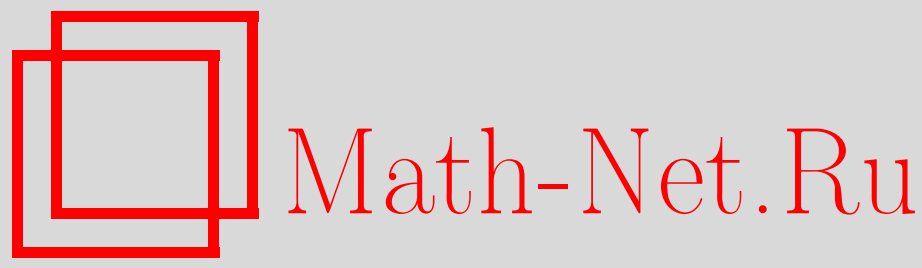

В. Б. Бобров, С. А. Тригер, Высокочастотное спектральное распределение энергии равновесного излучения в плазме, ТМФ, 2016, том 187, номер 1, 104-113

DOI: https://doi.org/10.4213/tmf8996

Использование Общероссийского математического портала Math-Net.Ru подразумевает, что вы прочитали и согласны с пользовательским соглашением http://www . mathnet.ru/rus/agreement

Параметры загрузки:

IP: 54.198 .187 .58

26 апреля 2023 г., 09:18:17

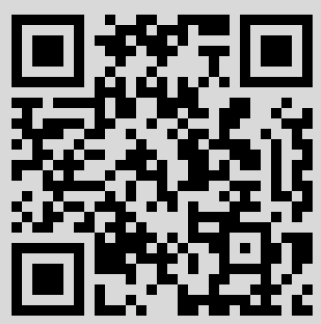




\title{
ВЫСОКОЧАСТОТНОЕ СПЕКТРАЛЬНОЕ РАСПРЕДЕЛЕНИЕ ЭНЕРГИИ РАВНОВЕСНОГО ИЗЛУЧЕНИЯ В ПЛАЗМЕ
}

\begin{abstract}
Установлено, что в области высоких частот отличие спектрального распределения энергии равновесного излучения в веществе от формулы Планка определяется мнимой частью поперечной диэлектрической проницаемости вещества. На этой основе показано, что в разреженной высокотемпературной полностью ионизованной нерелятивистской плазме высокочастотное спектральное распределение энергии равновесного излучения принципиально отличается от формулы Планка в силу степенного характера убывания по частоте, что обусловлено наличием вещества.
\end{abstract}

Ключевые слова: равновесное излучение, спектральное распределение энергии, полностью ионизованная плазма.

DOI: $10.4213 / \operatorname{tmf} 8996$

Спектральное распределение энергии равновесного излучения, установленное Планком [1], является фундаментальным соотношением, которое привело к становлению квантовой теории. Распределение Планка соответствует идеализированной модели абсолютно черного тела, представляющего собой свободную от вещества полость, заполненную излучением и ограниченную абсолютно поглощающим веществом. При этом предполагается, что излучение находится в термодинамическом равновесии с веществом, хотя эффекты взаимодействия фотонов с ограничивающим полость веществом не рассматриваются [2].

До последнего времени практическое использование распределения Планка было связано с рассмотрением макроскопического тела, находящегося в тепловом равновесии с окружающим его "черным" излучением [2]. В решении этой задачи, которая имеет непосредственное отношение к закону Кирхгофа, достигнуты большие успехи (см. подробности в трудах [3]-[5] и цитированную в них литературу). Однако

Настоящая работа выполнена при финансовой поддержке Российского научного фонда (грант № 14-19-01492).

* Объединенный институт высоких температур РАН, Москва, Россия.

E-mail: vic5907@mail.ru, satron@mail.ru 
вопросу о спектральном распределении энергии излучения в самом веществе, находящемся в состоянии равновесия, практически не уделялось внимания [6]-[8]. Решение этой задачи в основном ограничивалось анализом областей прозрачности при малых импульсах фотонов. Такой подход представляется заведомо ограниченным, так как из физических соображений ясно, что для установления термодинамического равновесия излучения в веществе необходимо учитывать эффекты поглощения излучения.

С другой стороны, вопрос об энергии электромагнитного поля в поглощающей среде в рамках электродинамики сплошных сред связан, как известно, с серьезными трудностями [9]. С формальной точки зрения проблема заключается в установлении соотношения между энергией электромагнитного поля и общим выражением для тепловых потерь в среде в нестационарных условиях, когда средние значения напряженностей электромагнитного поля отличны от нуля и изменяются во времени. Это и приводит в конечном итоге к рассмотрению только областей прозрачности [9], [10]. В такой ситуации решение задачи возможно для равновесного состояния среды в статическом электромагнитном поле или, в крайнем случае, при слабой зависимости электромагнитного поля от времени [9], [10]. Рассмотрение при использовании теории линейного отклика приводит к установлению определенных ограничений на электродинамические функции отклика, в том числе для диэлектрической проницаемости среды (см. подробнее работу [11]).

Однако случай равновесного поля (точнее, равновесной системы, представляющей собой совокупность электромагнитного поля и вещества (заряженных частиц)) является в этом смысле исключением. В такой системе тепловые потери отсутствуют, т. е. поглощение электромагнитного поля уравновешено его испусканием [12]. При этом средние значения напряженностей электромагнитного поля равны нулю.

Термодинамические свойства системы, состоящей из заряженных частиц и фотонов, находящихся в объеме $V$, полностью определяются ее термодинамическим потенциалом Гиббса $\Omega$ [13],

$$
\Omega\left(T, V,\left\{\gamma_{a}\right\}\right)=-T \ln Z\left(T, V,\left\{\gamma_{a}\right\}\right)
$$

где $T$ - температура рассматриваемой системы в энергетических единицах, а большая статистическая сумма $Z\left(T, V,\left\{\gamma_{a}\right\}\right)$ определяется равенством

$$
Z\left(T, V,\left\{\gamma_{a}\right\}\right)=\operatorname{Tr} \exp \left\{-\frac{1}{T}\left(\widehat{H}-\sum_{a} \gamma_{a} \widehat{N}_{a}\right)\right\}
$$

В этом выражении $\widehat{H}$ - гамильтониан системы в представлении вторичного квантования (здесь и далее мы ограничиваемся нерелятивистским приближением для заряженных частиц [14]),

$$
\widehat{H}=\widehat{H}_{\mathrm{part}}+\widehat{H}_{\mathrm{ph}},
$$

где $\widehat{H}_{\text {рart }}$ - гамильтониан взаимодействующих заряженных частиц в квантованном электромагнитном поле и $\widehat{H}_{\mathrm{ph}}$ - гамильтониан свободного поля излучения. 
Оператор $\widehat{H}_{\text {part }}$ имеет вид

$$
\begin{aligned}
\widehat{H}_{\text {part }}= & \sum_{a} \frac{\hbar^{2}}{2 m_{a}} \int d^{3} r\left(\nabla+\frac{i z_{a} e}{\hbar} \widehat{\mathbf{A}}(\mathbf{r})\right) \widehat{\Psi}_{a}^{+}(\mathbf{r})\left(\nabla-\frac{i z_{a} e}{\hbar} \widehat{\mathbf{A}}(\mathbf{r})\right) \widehat{\Psi}_{a}(\mathbf{r})- \\
& -\sum_{a} \int d^{3} r \widehat{\Psi}_{a}^{+}(\mathbf{r}) \hat{\mu}_{a} \widehat{\Psi}_{a}(\mathbf{r}) \operatorname{rot} \widehat{\mathbf{A}}(\mathbf{r})+\widehat{H}_{\mathrm{Coul}},
\end{aligned}
$$

где $\widehat{\Psi}_{a}^{+}(\mathbf{r})$ и $\widehat{\Psi}_{a}(\mathbf{r})$ - полевые операторы рождения и уничтожения для заряженных частиц сорта $a$, которые характеризуются массой $m_{a}$ и зарядом $z_{a} e$, оператором собственного магнитного момента $\hat{\mu}_{a}$ и химическим потенциалом $\gamma_{a}$, а оператор

$$
\widehat{N}_{a}=\int d^{3} r \widehat{\Psi}_{a}^{+}(\mathbf{r}) \widehat{\Psi}_{a}(\mathbf{r})
$$

есть оператор полного числа частиц сорта $a$. В формуле (4) $\widehat{H}_{\text {Coul }}$ - гамильтониан кулоновского взаимодействия заряженных частиц,

$$
\widehat{H}_{\text {Coul }}=\frac{1}{2} \sum_{a, b} \int d^{3} r_{1} d^{3} r_{2} u_{a b}\left(\left|\mathbf{r}_{1}-\mathbf{r}_{2}\right|\right) \widehat{\Psi}_{a}^{+}\left(\mathbf{r}_{1}\right) \widehat{\Psi}_{b}^{+}\left(\mathbf{r}_{2}\right) \widehat{\Psi}_{b}\left(\mathbf{r}_{2}\right) \widehat{\Psi}_{a}\left(\mathbf{r}_{1}\right),
$$

где $u_{a b}(r)=z_{a} z_{b} e^{2} / r-$ потенциал кулоновского взаимодействия заряженных частиц сортов $a$ и $b$.

Оператор $\widehat{H}_{\mathrm{ph}}$ задается как

$$
\widehat{H}_{\mathrm{ph}}=\sum_{\mathbf{k}, \lambda} \hbar \omega_{\mathbf{k}} \hat{c}_{\mathbf{k}, \lambda}^{+} \hat{c}_{\mathbf{k}, \lambda}, \quad \omega_{\mathbf{k}}=c|\mathbf{k}| .
$$

Здесь операторы рождения $\hat{c}_{\mathbf{k}, \lambda}^{+}$и уничтожения $\hat{c}_{\mathbf{k}, \lambda}$ для фотонов с импульсом $\hbar \mathbf{k}$ и поляризацией $\lambda=1,2$ удовлетворяют перестановочным соотношениям

$$
\left[\hat{c}_{\mathbf{k}, \lambda}, \hat{c}_{\mathbf{k}^{\prime}, \lambda^{\prime}}^{+}\right]=\delta_{\mathbf{k}, \mathbf{k}^{\prime}} \delta_{\lambda, \lambda^{\prime}}
$$

а $\widehat{\mathbf{A}}(\mathbf{r})$ - оператор векторного потенциала, соответствующий квантованному электромагнитному полю, который имеет вид

$$
\widehat{\mathbf{A}}(\mathbf{r})=c \sum_{\mathbf{k}, \lambda}\left(\frac{2 \pi \hbar}{\omega_{\mathbf{k}} V}\right)^{1 / 2}\left\{\mathbf{e}_{\mathbf{k}}^{(\lambda)} \hat{c}_{\mathbf{k}, \lambda} \exp (i \mathbf{k} \cdot \mathbf{r})+\mathbf{e}_{\mathbf{k}}^{(\lambda)^{*}} \hat{c}_{\mathbf{k}, \lambda}^{+} \exp (-i \mathbf{k} \cdot \mathbf{r})\right\},
$$

где $\mathbf{e}_{\mathbf{k}}^{(\lambda)}-$ векторы поляризации фотонов, удовлетворяющие условиям

$$
\mathbf{e}_{\mathbf{k}}^{(\lambda)} \cdot \mathbf{k}=0, \quad \sum_{\lambda=1}^{2} e_{\mathbf{k} \alpha}^{(\lambda)} e_{\mathbf{k} \beta}^{(\lambda) *}=\delta_{\alpha, \beta}-\frac{k_{\alpha} k_{\beta}}{k^{2}}
$$

Химический потенциал для фотонов, как обычно [2], считается равным нулю.

В рамках статистической квантовой электродинамики вычисление термодинамического потенциала Гиббса (1) основано на использовании функциональных методов теории возмущений по взаимодействию заряженных частиц с квантованным электромагнитным полем, так что величина $\Omega\left(T, V,\left\{\gamma_{a}\right\}\right)$ является функционалом от 
функций Грина для частиц и фотонов. При этом фотонная функция Грина однозначно определяется диэлектрической проницаемостью среды [13].

В соответствии с формулами (1)-(8) мы можем определить среднюю энергию рассматриваемой системы:

$$
E=\langle\widehat{H}\rangle=\left\langle\widehat{H}_{\mathrm{part}}\right\rangle+\left\langle\widehat{H}_{\mathrm{ph}}\right\rangle,
$$

где угловые скобки обозначают усреднение с большим каноническим распределением Гиббса. Оно характеризуется объемом $V$, который занимает рассматриваемая система, температурой $T$, одинаковой для фотонов и заряженных частиц, а также набором химических потенциалов $\left\{\gamma_{a}\right\}$ для заряженных частиц (см. соотношение (2)).

Далее мы ограничимся рассмотрением величины $E_{\mathrm{ph}} \equiv\left\langle\widehat{H}_{\mathrm{ph}}\right\rangle$, которую естественно считать средней энергией равновесного излучения в отличие от средней энергии взаимодействующих заряженных частиц в квантованном электромагнитном поле, определяемой как $E_{\text {part }} \equiv\left\langle\widehat{H}_{\text {part }}\right\rangle$. По аналогии с рассмотрением идеального газа фотонов средняя энергия равновесного излучения в веществе может быть представлена в виде [15]

$$
E_{\mathrm{ph}}=V \sum_{\lambda} \int \frac{d^{3} k}{(2 \pi)^{3}} \hbar \omega_{\mathbf{k}} f(\mathbf{k}, \lambda)=V \int_{0}^{\infty} d \omega \varepsilon_{\omega}\left(T,\left\{\gamma_{a}\right\}\right)
$$

где $f(\mathbf{k}, \lambda) \equiv\left\langle\hat{c}_{\mathbf{k}, \lambda}^{+} \hat{c}_{\mathbf{k}, \lambda}\right\rangle$ - точная равновесная функция распределения фотонов по импульсам. Очевидно, что спектральное распределение энергии излучения в веществе $\varepsilon_{\omega}\left(T,\left\{\gamma_{a}\right\}\right)$ зависит не только от частоты и температуры, как это имеет место в формуле Планка для идеального газа фотонов, но и от характеристик вещества, а именно от набора химических потенциалов заряженных частиц $\left\{\gamma_{a}\right\}$.

Как показано в работе [15], для спектрального распределения энергии излучения в веществе справедливо соотношение

$$
\varepsilon_{\omega}\left(T,\left\{\gamma_{a}\right\}\right)=\varepsilon_{\omega}^{(0)}(T)+\Delta \varepsilon_{\omega}\left(T,\left\{\gamma_{a}\right\}\right)
$$

где величина $\varepsilon_{\omega}^{(0)}(T)$ задается формулой Планка,

$$
\varepsilon_{\omega}^{(0)}(T)=\frac{\hbar}{\pi^{2} c^{3}} \frac{\omega^{3}}{\exp (\hbar \omega / T)-1},
$$

а функция $\Delta \varepsilon_{\omega}\left(T,\left\{\gamma_{a}\right\}\right)-$ равенством

$$
\Delta \varepsilon_{\omega}\left(T,\left\{\gamma_{a}\right\}\right)=\frac{\hbar \omega^{3}}{\pi^{2} c^{3}} \operatorname{cth}\left(\frac{\hbar \omega}{2 T}\right)\left(\frac{c^{5}}{\pi \omega} \int_{0}^{\infty} d k k^{4} \frac{\operatorname{Im} \varepsilon^{\operatorname{tr}}(k, \omega)}{\left|\varepsilon^{\operatorname{tr}}(k, \omega) \omega^{2}-c^{2} k^{2}\right|^{2}}-\frac{1}{2}\right) .
$$

В результате отличие спектрального распределения энергии излучения в веществе от формулы Планка (3) полностью определяется поперечной диэлектрической проницаемостью $\varepsilon^{\operatorname{tr}}(k, \omega)$ рассматриваемой системы. При этом соотношение (4) справедливо только для однородной и изотропной системы, линейные электромагнитные свойства которой однозначно определяются продольной $\varepsilon^{\ell}(k, \omega)$ и поперечной $\varepsilon^{\operatorname{tr}}(k, \omega)$ диэлектрическими проницаемостями [16]. 
Как следует из выражения (13), принципиальное значение имеет учет не только частотной, но и пространственной дисперсии поперечной диэлектрической проницаемости $\varepsilon^{\operatorname{tr}}(k, \omega)$, особенно в области больших волновых векторов $k$. Для обеспечения сходимости интеграла в (13) при фиксированной частоте $\omega$ должно выполняться условие

$$
\lim _{k \rightarrow \infty} \operatorname{Im} \varepsilon^{\operatorname{tr}}(k, \omega)=0 .
$$

Это означает, что при вычислении функции $\varepsilon^{\operatorname{tr}}(k, \omega)$ необходимо последовательно учитывать квантовые эффекты. Действительно, при рассмотрении квантовой идеальной (бесстолкновительной) плазмы в области больших волновых векторов $k$ имеет место соотношение $\operatorname{Im} \varepsilon^{\operatorname{tr}}(k, \omega) \sim \exp \left(-\hbar^{2} k^{2} / 8 T m_{a}\right)$ (см. подробности в работах [17], [18]). Обратим внимание, что требование $(14)$ для $\operatorname{Im} \varepsilon^{\operatorname{tr}}(k, \omega)$ должно выполняться и при вычислении потерь энергии пробной заряженной частицы, проходящей через плазму [16], [19]. Таким образом, мы можем рассматривать условие (14) как общее свойство функции $\operatorname{Im} \varepsilon^{\operatorname{tr}}(k, \omega)$.

Общее выражение для функции $\varepsilon^{\operatorname{tr}}(k, \omega)$ для рассматриваемой системы можно найти в рамках теории линейного отклика, оно имеет вид [14]

$$
\varepsilon^{\operatorname{tr}}(k, \omega)=1-\frac{\omega_{\mathrm{p}}^{2}}{\omega^{2}}-\frac{4 \pi}{\omega^{2}} \Phi^{\mathrm{R}}(k, \omega), \quad \Phi^{\mathrm{R}}(k, \omega)=\frac{1}{2}\left(\delta_{\alpha, \beta}-\frac{k_{\alpha} k_{\beta}}{k^{2}}\right) \Phi_{\alpha \beta}^{\mathrm{R}}(\mathbf{k}, \omega),
$$

где $\omega_{\mathrm{p}}=\left(\sum_{a} 4 \pi z_{a}^{2} e^{2} n_{a} / m_{a}\right)^{1 / 2}$ - плазменная частота, $n_{a}-$ плотность числа частиц сорта $a, \Phi_{\alpha \beta}^{\mathrm{R}}(\mathbf{k}, \omega)$ - запаздывающая тензорная функция Грина "ток-ток", которая равна

$$
\Phi_{\alpha \beta}^{\mathrm{R}}(\mathbf{k}, \omega)=\int d^{3} r \exp (-i \mathbf{k} \cdot \mathbf{r}) \int_{0}^{\infty} d t \exp (i \omega t) \Phi_{\alpha \beta}^{\mathrm{R}}(\mathbf{r}, t),
$$

где

$$
\Phi_{\alpha \beta}^{\mathrm{R}}\left(\mathbf{r}_{1}-\mathbf{r}_{2}, t\right)=-\frac{i}{\hbar}\left\langle\left[\widehat{J}_{\alpha}\left(\mathbf{r}_{1}, t\right), \widehat{J}_{\beta}\left(\mathbf{r}_{2}, 0\right)\right]\right\rangle .
$$

Здесь $\widehat{\mathbf{J}}(\mathbf{r}, t)$ - векторный оператор плотности тока заряженных частиц при наличии электромагнитного поля в представлении Гейзенберга,

$$
\begin{aligned}
\widehat{\mathbf{J}}(\mathbf{r})= & \sum_{a} \frac{i \hbar z_{a} e}{2 m_{a}}\left\{\left[\left(\nabla+\frac{i z_{a} e}{c} \widehat{\mathbf{A}}(\mathbf{r})\right) \widehat{\Psi}_{a}^{+}(\mathbf{r})\right] \widehat{\Psi}_{a}(\mathbf{r})-\right. \\
& \left.-\widehat{\Psi}_{a}^{+}(\mathbf{r})\left[\left(\nabla-\frac{i z_{a} e}{c} \widehat{\mathbf{A}}(\mathbf{r})\right) \widehat{\Psi}_{a}(\mathbf{r})\right]\right\}+\operatorname{rot} \sum_{a} \Psi_{a}^{+}(\mathbf{r}) \hat{\mu}_{a} \widehat{\Psi}_{a}(\mathbf{r}) .
\end{aligned}
$$

Как следует из соотношений (15)-(18), в общем случае вычисление поперечной диэлектрической проницаемости $\varepsilon^{\operatorname{tr}}(k, \omega)$ рассматриваемой системы является весьма сложной задачей. Это в еще большей степени относится к вычислению интеграла в (13) для определения величины $\Delta \varepsilon_{\omega}\left(T,\left\{\gamma_{a}\right\}\right)$. Однако при рассмотрении высокочастотного предела $\omega \rightarrow \infty$ соотношение (13) можно существенно упростить. Дело в том, что согласно (15) при фиксированном значении волнового вектора $k$ мы имеем (см. подробности в работе [13])

$$
\lim _{\omega \rightarrow \infty} \operatorname{Re} \varepsilon^{\operatorname{tr}}(k, \omega)=1, \quad \lim _{\omega \rightarrow \infty} \operatorname{Im} \varepsilon^{\operatorname{tr}}(k, \omega)=0 .
$$


Рассмотрим функцию

$$
F(\omega)=\frac{c^{5}}{\pi \omega^{5}} \int_{0}^{\infty} d k k^{4} \frac{\operatorname{Im} \varepsilon^{\operatorname{tr}}(k, \omega)}{\left|\varepsilon^{\operatorname{tr}}(k, \omega)-c^{2} k^{2} / \omega^{2}\right|^{2}},
$$

определяющую величину $\Delta \varepsilon_{\omega}\left(T,\left\{\gamma_{a}\right\}\right)$ (см. формулу (13)). Эту функцию в пределе $\omega \rightarrow \infty$ запишем в виде

$$
\left.F(\omega)\right|_{\omega \rightarrow \infty}=F(\infty)+\Delta F(\omega), \quad F(\infty)=\lim _{\omega \rightarrow \infty} F(\omega), \quad \lim _{\omega \rightarrow \infty} \Delta F(\omega)=0 .
$$

Чтобы вычислить величину $F(\infty)$, необходимо учесть, что согласно (19)

$$
\left.\left(\frac{\operatorname{Im} \varepsilon^{\operatorname{tr}}(k, \omega)}{\left(\operatorname{Re} \varepsilon^{\operatorname{tr}}(k, \omega)-c^{2} k^{2} / \omega^{2}\right)^{2}+\left(\operatorname{Im} \varepsilon^{\operatorname{tr}}(k, \omega)\right)^{2}}\right)\right|_{\omega \rightarrow \infty}=\pi \delta\left(1-\frac{c^{2} k^{2}}{\omega^{2}}\right)
$$

где $\delta(x)$ - дельта-функция Дирака. Тогда с учетом (22) нетрудно убедиться, что

$$
F(\infty)=\lim _{\omega \rightarrow \infty} F(\omega)=\frac{1}{2}
$$

В свою очередь для функции $\Delta F(\omega)$, учитывая соотношения $(19)$, в пределе $\omega \rightarrow \infty$ можно записать

$$
\left.\Delta F(\omega)\right|_{\omega \rightarrow \infty}=\left.\frac{c^{5}}{\pi \omega^{5}}\left(\int_{0}^{\infty} d k k^{4} \operatorname{Im} \varepsilon^{\operatorname{tr}}(k, \omega)\right)\right|_{\omega \rightarrow \infty}
$$

Подставляя формулы (20)-(24) в (13), находим

$$
\left.\Delta \varepsilon_{\omega}\left(T,\left\{\gamma_{a}\right\}\right)\right|_{\omega \rightarrow \infty}=\left.\frac{\hbar c^{2}}{\pi^{3} \omega^{2}}\left(\int_{0}^{\infty} d k k^{4} \operatorname{Im} \varepsilon^{\operatorname{tr}}(k, \omega)\right)\right|_{\omega \rightarrow \infty} .
$$

Таким образом, появляется возможность решить вопрос о степени влияния вещества на спектральное распределение энергии равновесного излучения. Дело в том, что спектральное распределение "черного" излучения согласно формуле Планка (12) характеризуется экспоненциальным убыванием в пределе $\omega \rightarrow \infty$. В то же время согласно (25) высока вероятность того, что величина $\Delta \varepsilon_{\omega}\left(T,\left\{\gamma_{a}\right\}\right)$ убывает в пределе $\omega \rightarrow \infty$ по степенному закону. В случае справедливости такого утверждения в пределе $\omega \rightarrow \infty$ спектральное распределение энергии равновесного излучения в веществе принципиально отличается от формулы Планка. Однако в настоящее время установить общую закономерность в частотной зависимости величины интеграла в (25) не представляется возможным.

Чтобы упростить наше рассмотрение, учтем, что постоянная тонкой структуры $\alpha=e^{2} / \hbar c \cong 1 / 137$, которая характеризует силу взаимодействия между электрическими зарядами и фотонами, является малым параметром. Это обстоятельство составляет основу использования в квантовой статистической электродинамике теории возмущений (диаграммной техники), связанной с представлением средних значений физических величин в виде рядов по степеням $\alpha$ [13]. В частности, вывод соотношений (11)-(13) для спектральной плотности равновесного излучения в веществе основан на результатах диаграммной техники теории возмущений для фотонной функции Грина [15]. 
При вычислении поперечной диэлектрической проницаемости $\varepsilon^{\operatorname{tr}}(k, \omega)$ мы ограничимся рассмотрением "нулевого" приближения по параметру $\alpha$. Другими словами, далее мы будем рассматривать функцию $\varepsilon^{\operatorname{tr}}(k, \omega)$, заданную равенствами $(15)-(18)$, для системы заряженных частиц, взаимодействующих по закону Кулона, пренебрегая их взаимодействием с фотонами. В этом случае, как нетрудно убедиться, функцию $\Phi_{\alpha \beta}^{\mathrm{R}}(\mathbf{k}, \omega)$ можно представить в виде

$$
\Phi_{\alpha \beta}^{\mathrm{R}}(\mathbf{k}, \omega)=\Phi_{\alpha \beta}^{(\mathrm{dd})}(\mathbf{k}, \omega)+\Phi_{\alpha \beta}^{(\mathrm{pp})}(\mathbf{k}, \omega)
$$

где функция $\Phi_{\alpha \beta}^{(\mathrm{dd})}(\mathbf{k}, \omega)$ отвечает диамагнитной части, а функция $\Phi_{\alpha \beta}^{(\mathrm{pp})}(\mathbf{k}, \omega)$ - парамагнитной части функции Грина $\Phi_{\alpha \beta}^{\mathrm{R}}(\mathbf{k}, \omega)$. Функции $\Phi_{\alpha \beta}^{(\mathrm{dd})}(\mathbf{k}, \omega)$ и $\Phi_{\alpha \beta}^{(\mathrm{pp})}(\mathbf{k}, \omega)$ определяются соотношениями (16), (17) с точностью до замены оператора $\widehat{\mathbf{J}}(\mathbf{r})(18)$ на операторы $\widehat{\mathbf{J}}^{(\mathrm{d})}(\mathbf{r})$ и $\widehat{\mathbf{J}}^{(\mathrm{p})}(\mathbf{r})$ соответственно, где

$$
\begin{aligned}
& \widehat{\mathbf{J}}^{(\mathrm{d})}(\mathbf{r})=\sum_{a} \frac{i \hbar z_{a} e}{2 m_{a}}\left\{\left(\nabla \widehat{\Psi}_{a}^{+}(\mathbf{r})\right) \widehat{\Psi}_{a}(\mathbf{r})-\widehat{\Psi}_{a}^{+}(\mathbf{r})\left(\nabla \widehat{\Psi}_{a}(\mathbf{r})\right)\right\} \\
& \widehat{\mathbf{J}}^{(\mathrm{p})}(\mathbf{r})=\operatorname{rot} \sum_{a} \widehat{\Psi}_{a}^{+}(\mathbf{r}) \hat{\mu}_{a} \widehat{\Psi}_{a}(\mathbf{r}) .
\end{aligned}
$$

Кроме того, обе функции $\Phi_{\alpha \beta}^{(\mathrm{dd})}(\mathbf{k}, \omega)$ и $\Phi_{\alpha \beta}^{(\mathrm{pp})}(\mathbf{k}, \omega)$ являются неприводимыми по одной линии кулоновского взаимодействия заряженных частиц в " $k$-канале". При этом функция $\Phi_{\alpha \beta}^{(\mathrm{pp})}(\mathbf{k}, \omega)$ также является неприводимой по двум линиям кулоновского взаимодействия [17].

Чтобы исключить необходимость учета эффектов кулоновского взаимодействия, приводящих к образованию связанных состояний заряженных частиц, рассмотрим далее слабонеидеальную полностью ионизованную плазму, удовлетворяющую условиям

$$
\frac{m_{e} e^{4}}{\hbar^{2}} \ll T \ll m_{e} c^{2}, \quad \frac{z_{a}^{2} e^{2}}{\left\langle r_{a}\right\rangle\left\langle k_{a}\right\rangle} \ll 1,
$$

где $\left\langle r_{a}\right\rangle=\left(4 \pi n_{a} / 3\right)^{-1 / 3}$ - среднее расстояние между частицами, $\left\langle k_{a}\right\rangle$ - средняя кинетическая энергия, приходящаяся на одну частицу сорта $a$ [20]. В этом случае при вычислении функций $\Phi_{\alpha \beta}^{(\mathrm{dd})}(\mathbf{k}, \omega)$ и $\Phi_{\alpha \beta}^{(\mathrm{pp})}(\mathbf{k}, \omega)$ можно пренебречь кулоновским взаимодействием, что отвечает рассмотрению так называемой бесстолкновительной плазмы (далее мы отмечаем этот факт нижним индексом 0), см. подробности в работах [17], [18]:

$$
\varepsilon_{0}^{\operatorname{tr}}(k, \omega)=1-\frac{\omega_{\mathrm{p}}^{2}}{\omega^{2}}-\frac{4 \pi}{\omega^{2}}\left(\Phi_{0}^{(\mathrm{dd})}(k, \omega)+\Phi_{0}^{(\mathrm{pp})}(k, \omega)\right),
$$

где

$$
\begin{aligned}
\Phi_{0}^{(\mathrm{dd})}(k, \omega)= & \frac{1}{2} \sum_{a}\left(2 s_{a}+1\right) \frac{z_{a}^{2} e^{2} \hbar^{2}}{m_{a}^{2}} \times \\
& \times \int \frac{d^{3} p}{(2 \pi)^{3}}\left(p^{2}-\frac{(\mathbf{p} \cdot \mathbf{k})^{2}}{k^{2}}\right) \frac{f_{a}(\mathbf{p}-\mathbf{k} / 2)-f_{a}(\mathbf{p}+\mathbf{k} / 2)}{\hbar \omega+\epsilon_{a}(\mathbf{p}-\mathbf{k} / 2)-\epsilon_{a}(\mathbf{p}+\mathbf{k} / 2)+i 0},
\end{aligned}
$$




$$
\begin{aligned}
\Phi_{0}^{(\mathrm{pp})}(k, \omega)= & k^{2} \sum_{a} \frac{\left(2 s_{a}+1\right) s_{a}\left(s_{a}+1\right)}{3}\left(\frac{\mu_{a} c}{s_{a}}\right)^{2} \times \\
& \times \int \frac{d^{3} p}{(2 \pi)^{3}} \frac{f_{a}(\mathbf{p}-\mathbf{k} / 2)-f_{a}(\mathbf{p}+\mathbf{k} / 2)}{\hbar \omega+\epsilon_{a}(\mathbf{p}-\mathbf{k} / 2)-\epsilon_{a}(\mathbf{p}+\mathbf{k} / 2)+i 0} .
\end{aligned}
$$

Здесь $\epsilon_{a}(p)=\hbar^{2} p^{2} / 2 m_{a}$ - энергия частицы, $f_{a}(p)$ - функция распределения по импульсам для частиц сорта $a$, которая определяется распределениями Ферми-Дирака или Бозе-Эйнштейна в зависимости от спина частицы $s_{a}$. Величина химического потенциала $\gamma_{a}$ при заданной температуре определяется плотностью числа частиц $n_{a}$ согласно условию

$$
n_{a}=\left(2 s_{a}+1\right) \int \frac{d^{3} p}{(2 \pi)^{3}} f_{a}(p)
$$

При рассмотрении разреженной плазмы, удовлетворяющей помимо (29) условиям $n_{a} \Lambda_{a}^{3} \ll 1$, где $\Lambda_{a}=\left(2 \pi \hbar^{2} / m_{a} T\right)^{1 / 2}$ - тепловая длина волны де Бройля, из условия (33) находим

$$
f_{a}(p) \cong \frac{n_{a} \Lambda_{a}^{3}}{2 s_{a}+1} \exp \left(-\frac{\epsilon_{a}(p)}{T}\right), \quad \frac{\gamma_{a}}{T} \cong \ln \frac{n_{a} \Lambda_{a}^{3}}{2 s_{a}+1}<0, \quad \frac{\left|\gamma_{a}\right|}{T} \gg 1
$$

В этом случае из соотношений (30)-(34) непосредственно следует, что

$$
\operatorname{Im} \varepsilon_{0}^{\operatorname{tr}}(k, \omega)=\operatorname{Im} \varepsilon_{0}^{(\mathrm{dd})}(k, \omega)+\operatorname{Im} \varepsilon_{0}^{(\mathrm{pp})}(k, \omega),
$$

где

$$
\begin{aligned}
\operatorname{Im} \varepsilon_{0}^{(\mathrm{dd})}(k, \omega)= & \sum_{a} \frac{\omega_{a}^{2}}{\omega^{2}} \frac{\left(2 \pi m_{a} T\right)^{1 / 2}}{\hbar k} \operatorname{sh}\left(\frac{\hbar \omega}{2 T}\right) \exp \left(-\frac{m_{a} \omega^{2}}{2 T k^{2}}-\frac{\hbar^{2} k^{2}}{8 m_{a} T}\right), \\
\operatorname{Im} \varepsilon_{0}^{(\mathrm{pp})}(k, \omega)= & \frac{2 \pi}{3} \sum_{a} s_{a}\left(s_{a}+1\right)\left(\frac{\mu_{a} m_{a} c}{\hbar z_{a} e s_{a}}\right)^{2} \frac{\omega_{a}^{2}}{\omega^{2}} \frac{\hbar k}{\left(2 \pi m_{a} T\right)^{1 / 2}} \times \\
& \times \operatorname{sh}\left(\frac{\hbar \omega}{2 T}\right) \exp \left(-\frac{m_{a} \omega^{2}}{2 T k^{2}}-\frac{\hbar^{2} k^{2}}{8 m_{a} T}\right)
\end{aligned}
$$

здесь $\omega_{a}=\left(4 \pi z_{a}^{2} e^{2} n_{a} / m_{a}\right)^{1 / 2}$ - плазменная частота для частиц сорта $a$.

Подставляя формулы (35)-(37) в (25) и учитывая, что

$$
\begin{gathered}
\int_{0}^{\infty} d x x^{\nu-1} \exp \left(-\frac{\beta}{x}-\gamma x\right)=2\left(\frac{\beta}{\gamma}\right)^{\nu / 2} K_{\nu}(2 \sqrt{\beta \gamma}), \\
\left.K_{\nu}(x)\right|_{x \rightarrow \infty}=\left(\frac{\pi}{2 x}\right)^{1 / 2} \exp (-x),
\end{gathered}
$$


где $K_{\nu}(x)$ - модифицированная функция Бесселя второго рода (или функция Макдональда) [21], находим, что

$$
\left.\Delta \varepsilon_{\omega}\left(T,\left\{\gamma_{a}\right\}\right)\right|_{\omega \rightarrow \infty}=\Delta \varepsilon_{\omega}^{(\mathrm{dd})}+\Delta \varepsilon_{\omega}^{(\mathrm{pp})},
$$

где

$$
\begin{aligned}
& \Delta \varepsilon_{\omega}^{(\mathrm{dd})}=\frac{2 \sqrt{2} T}{\pi c^{3}} \sum_{a} \omega_{a}^{2}\left(\frac{m_{a} c^{2}}{\hbar \omega}\right)^{5 / 2}, \\
& \Delta \varepsilon_{\omega}^{(\mathrm{pp})}=\frac{8}{\pi^{2}} \sum_{a} s_{a}\left(s_{a}+1\right) \frac{m_{a} \omega_{a}^{2}}{c}\left(\frac{\mu_{a} m_{a} c}{\hbar z_{a} e s_{a}}\right)^{2}\left(\frac{m_{a} c^{2}}{\hbar \omega}\right)^{3 / 2} .
\end{aligned}
$$

Обратим внимание, что в области высоких частот величина $\left.\Delta \varepsilon_{\omega}\left(T,\left\{\gamma_{a}\right\}\right)\right|_{\omega \rightarrow \infty}$ полностью определяется "парамагнитной" функцией $\Delta \varepsilon_{\omega}^{(\mathrm{pp})}(30)$.

Таким образом, согласно соотношениям (11), (12), (39)-(41), как и предполагалось выше, высокочастотное спектральное распределение энергии излучения в идеальной (бесстолкновительной) квазиклассической плазме полностью определяется наличием вещества,

$$
\left.\varepsilon_{\omega}\left(T,\left\{\gamma_{a}\right\}\right)\right|_{\omega \rightarrow \infty}=\frac{8}{\pi^{2}} \sum_{a} s_{a}\left(s_{a}+1\right) \frac{m_{a} \omega_{a}^{2}}{c}\left(\frac{\mu_{a} m_{a} c}{\hbar z_{a} e s_{a}}\right)^{2}\left(\frac{m_{a} c^{2}}{\hbar \omega}\right)^{3 / 2},
$$

и принципиально отличается от формулы Планка (12) в силу степенной зависимости. Отметим, что соотношение (42) является асимптотическим. Чтобы установить отличие спектрального распределения энергии равновесного излучения в плазме от формулы Планка при конечных частотах $\omega$, необходимо проведение численных расчетов.

В заключение подчеркнем, что полученный результат имеет место для равновесной однородной и изотропной системы, представляющей собой взаимодействующие между собой вещество и электромагнитное поле, которые находятся в состоянии термодинамического равновесия с термостатом при заданной температуре $T$. При этом в качестве вещества рассматривается разреженная полностью ионизованная плазма. Соответствующее равновесное состояние рассматриваемой системы характеризуется определенной полной средней энергией. Из этой энергии можно выделить часть, отвечающую энергии электромагнитного поля, при вычислении которой необходимо учитывать наличие вещества в рассматриваемой системе. Подобная постановка задачи соответствует также рассмотрению черного излучения с той лишь разницей, что все вещество сосредоточено в термостате с абсолютно поглощающими стенками. При стремлении плотности вещества к нулю найденное нами спектральное распределение энергии излучения переходит в распределение Планка с температурой термостата.

Отметим, что вопрос об измерении спектральной плотности энергии излучения должен быть исследован отдельно на основе анализа распространения электромагнитного излучения в неоднородной среде, что выходит за рамки представленной работы.

Благодарности. Авторы благодарны А. М. Игнатову и А. А. Рухадзе за полезные обсуждения. 


\section{Список литературы}

[1] M. Planck, Ann. Phys., 309:3 (1901), 553-563.

[2] Л. Д. Ландау, Е. М. Лифшиц, Теоретическая физика, т. 5: Статистическая физика. Часть 1, Наука, М., 1976.

[3] М. Л. Левин, С. М. Рытов, Теория равновесных тепловых флуктуаций в электродинамике, Наука, М., 1967.

[4] А. И. Волокитин, Б. Н. Дж. Перссон, УФН, 177:9 (2007), 921-951.

[5] Е. А. Виноградов, И. А. Дорофеев, УФН, 179:5 (2009), 449-485.

[6] V.B. Bobrov, J. Phys.: Cond. Matt., 2:31 (1990), 6695-6698.

[7] В. Б. Бобров, ТМФ, 88:1 (1991), 141-145.

[8] S. A. Trigger, Phys. Lett. A, 370:5-6 (2007), 365-369, arXiv: cond-mat/0703572.

[9] Л. Д. Ландау, Е. М. Лифшиц, Теоретическая физика, т. 8: Электродинамика сплошнъх сред, Наука, М., 1982.

[10] В. М. Агранович, В. Л. Гинзбург, Электродинамика с учетом пространственной дисперсии и теория экситонов, Наука, М., 1964.

[11] Д. А. Киржниц, УФН, 152:3 (1987), 399-422.

[12] Ю. С. Бараш, В. Л. Гинзбург, УФН, 116:1 (1975), 5-40.

[13] Е. С. Фрадкин, Тр. ФИАН, 29 (1965), 7-138.

[14] А.И. Ахиезер, С.В. Пелетминский, Методь статистической физики, Наука, М., 1977.

[15] В. Б. Бобров, И. М. Соколов, С. А. Тригер, Писъма в ЖКЭТФ, 101:5 (2015), 326-329.

[16] В.П. Силин, А.А. Рухадзе, Электромагнитные свойства плазмы и плазмоподобных сред, Либроком, М., 2013.

[17] V.B. Bobrov, Physica A, 187:3-4 (1992), 603-624.

[18] М. В. Кузелев, А. А. Рухадзе, УФН, 169:6 (1999), 687-689.

[19] Д. А. Киржниц, Писъма в ЖКЭТФ, 46:6 (1987), 244-246.

[20] В.-Д. Крефт, Д. Кремп, В. Эбелинг, Г. Рёпке, Квантовая статистика систем заряженных частии, Мир, М., 1988.

[21] И. С. Градштейн, И.М. Рыжик, Таблицы интегралов, сумм, рядов и произведений, Физматгиз, М., 1963.

Поступила в редакцию 20.06.2015, после доработки 16.08.2015 